\title{
The complexity of constructing gerechte designs
}

\author{
E. R. Vaughan \\ School of Mathematical Sciences, Queen Mary, University of London, UK \\ e.vaughan@qmul.ac.uk
}

Submitted: Nov 20, 2007; Accepted: Jan 20, 2009; Published: Jan 30, 2009

Mathematics Subject Classification: 05B15, 68Q17

\begin{abstract}
Gerechte designs are a specialisation of latin squares. A gerechte design is an $n \times n$ array containing the symbols $\{1, \ldots, n\}$, together with a partition of the cells of the array into $n$ regions of $n$ cells each. The entries in the cells are required to be such that each row, column and region contains each symbol exactly once. We show that the problem of deciding if a gerechte design exists for a given partition of the cells is NP-complete. It follows that there is no polynomial time algorithm for finding gerechte designs with specified partitions unless $\mathrm{P}=\mathrm{NP}$.
\end{abstract}

\section{Introduction}

Gerechte designs were introduced by W. U. Behrens in 1956 [2] as a specialisation of latin squares. A gerechte skeleton of order $n$ is an $n \times n$ array whose $n^{2}$ cells are partitioned into $n$ regions containing $n$ cells each. A gerechte design of order $n$ consists of a gerechte skeleton of order $n$, together with an assignment of a symbol from the set $\{1, \ldots, n\}$ to each cell, such that each symbol occurs once in each row, once in each column, and once in each region of the array. We call such an assignment of symbols to cells a completion.

Not all gerechte skeletons have completions. For example, suppose we have a skeleton in which one of the regions consists of all the cells in a row except for the cell in column $n$, and a cell in column $n$ in a different row. Clearly a skeleton that contains such a region cannot have a completion.

Bailey, Cameron and Connelly in their article on sudoku [1] asked what is the computational complexity of determining if a given gerechte skeleton has a completion? This question was also presented at the problem session of the CGCS Luminy conference in May 2007 [3]. We prove the following:

Theorem 1. The problem of determining if a gerechte skeleton has a completion is NPcomplete. 
It follows that the search problem of finding a completion for a given gerechte skeleton is NP-hard. In fact we will show that the search problem is in a sense "no harder" than the decision problem, as the search problem is Turing-reducible to the decision problem. (Throughout, we will use the definitions of Garey and Johnson [7].)

It should be noted that this problem is quite different to that of completing a partial latin square, which was shown to be NP-complete by Colbourn [4]. The input to the problem is simply the gerechte skeleton; there is no partial assignment of symbols to cells. But as the problem is a special case of the problem of completing a partially filled gerechte skeleton, we can restate the result as:

Theorem 2. The problem of determining if a partially filled gerechte skeleton has a completion is NP-complete, even if the number of filled cells is zero.

In this case "completion" means an assignment of symbols to cells that agrees with the partial filling, and satisfies the previous definition of completion.

\section{Edge-colouring of graphs}

To prove the NP-completeness of our problem, we use a polynomial transformation from the following problem, shown to be NP-complete by Holyer [8].

Problem. Given a cubic (3-regular) graph, is the chromatic index 3?

The chromatic index of a graph $G$ is the minimum number of colours required to colour the edges of $G$ such that adjacent edges are coloured differently. By Vizing's Theorem [5], all cubic graphs have chromatic index either 3 or 4 .

We will now prove lemma 4, which will be needed later. The lemma is a consequence of the following theorem.

Theorem 3. (König, 1916 [5]) Let $G$ be a bipartite graph. Then the chromatic index of $G$ is equal to the maximum vertex degree of $G$.

We define a partial latin square of order $n$ to be an $n \times n$ array, containing some empty cells and some cells filled with symbols from $\{1, \ldots, n\}$ with the condition that no symbol occurs more than once in any row or column. We say that a partial latin square is uniform if each row and column has the same subset of $\{1, \ldots, n\}$ as its entries.

Lemma 4. Suppose $P$ is a uniform partial latin square of order $n$ with exactly $k$ blank entries in each row and column, where $0 \leq k \leq n$. Then $P$ can always be completed to a latin square.

Proof. If there are no empty cells then we are done, otherwise let us construct a bipartite graph $G$ as follows. We create a vertex for each row and column of $P$, which we label $r_{1}, \ldots, r_{n}$ and $c_{1}, \ldots, c_{n}$. We add an edge for every blank cell of the partial latin square: if the cell in row $i$, column $j$, is blank, we add an edge between $r_{i}$ and $c_{j}$. So $G$ is bipartite with bipartition $\left\{\left\{r_{1}, \ldots, r_{n}\right\},\left\{c_{1}, \ldots, c_{n}\right\}\right\}$, and $k$-regular, so by König's theorem there 


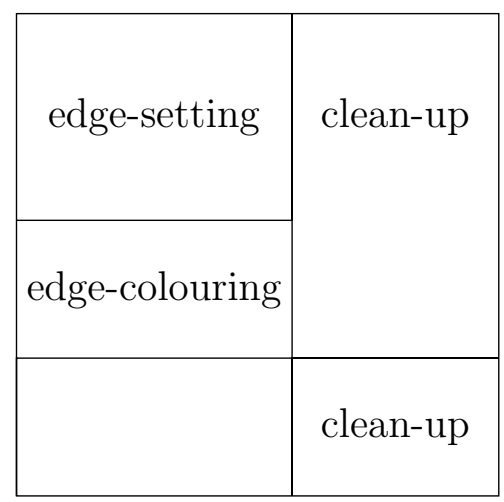

Figure 1: Layout of $S(G)$

is a $k$-colouring of its edges. We identify the $k$ colours with the $k$ symbols missing from each row and column. Then the empty cells in the array can be filled according to the colours of their corresponding edges in $G$.

\section{Transformation from edge-colouring}

Let $G$ be a cubic graph that has $2 m / 3$ vertices and $m$ edges (we will allow $G$ to have parallel edges, but not loops). We will give a construction of a gerechte skeleton of order $7 m$, which we will call $S(G)$, and we will show that $S(G)$ has a completion if and only if the chromatic index of $G$ is 3 .

Suppose (for a moment) we had a gerechte skeleton whose regions were precisely the rows of the array. It would be easy to complete, as any latin square of the appropriate order would be a completion. We could describe this skeleton by saying that each cell is in the region numbered similarly to its row. In our $S(G)$, most of the cells will be numbered similarly to their rows. In fact, in each row there will be at most three cells that are not. An equivalent way of saying this is that for each region there is a row that contains all but at most three of its cells.

$S(G)$ will be of order $n$, where $n=7 m$. It will be helpful to imagine $S(G)$ partitioned into a number of areas that will serve different purposes. These areas are depicted in figure 1 (which is drawn to scale). Now for all regions $j$, where $1 \leq j \leq n$, all but at most three of the cells will be in row $j$. The ones that are not will be referred to as exceptional cells. So in order to describe the partition of the array of $S(G)$ into $n$ regions it will suffice to describe the exceptional cells. All unexceptional cells will be understood to be in the region numbered similarly to their row.

Now in $S(G)$ rows $j$, where $3 m+1 \leq j \leq n$, will each contain three exceptional cells belonging to region $j-1$. The situation will be slightly different for the first $3 m$ rows. The first $3 m$ rows of the array contain the "edge-setting" area. Now $G$ must have at least 3 edges, so the number of rows in the edge-setting area is at least 9 . The exceptional cells in the first 9 rows will be laid out as shown in figure 2 (where the first 9 rows and 


\begin{tabular}{|l|l|l|l|l|l|l|l|l|l|l|l|}
\hline $\mathrm{n}$ & & $\mathrm{n}$ & & & & & & & & & \\
\hline & $\mathrm{n}$ & & & & & & & & & & \\
\hline 1 & & 1 & 2 & & & & & & & & \\
\hline & & & & 3 & & 3 & & & & & \\
\hline & & & & & 3 & & & & & & \\
\hline & & & & 4 & & 4 & 5 & & & & \\
\hline & & & & & & & & 6 & & 6 & \\
\hline & & & & & & & & & 6 & & \\
\hline & & & & & & & & 7 & & 7 & 8 \\
\hline
\end{tabular}

Figure 2: The first 9 rows of $S(G)$

12 columns of $S(G)$ are shown). In this diagram exceptional cells have been indicated by a label saying which region they belong to. The unexceptional cells have been left unlabelled. The first $3 m$ rows of $S(G)$ will be laid out in this manner, following the pattern established in the first 9 rows.

Below the edge-setting rows, the situation is simpler. Exceptional cells in row $j$ always belong to region $j-1$, so it will not be necessary to explicitly say which region exceptional cells belong to. It will suffice to specify the locations of the exceptional cells. In figure 4, we give the layout of $S\left(K_{4}\right)$. Black cells indicate exceptional cells.

The "edge-colouring" area is the section of the array where we recreate the graph edge-colouring problem. The edge-colouring area has $2 m$ rows, which is three for each vertex of $G$. Suppose the vertices of $G$ are labelled $\{1, \ldots, 2 m / 3\}$. Then vertex $j$ is allocated rows $3 m+3 j-2,3 m+3 j-1$, and $3 m+3 j$.

Suppose the edges are labelled $\{1, \ldots, m\}$. Then we can label the ends of the edges $\{1, \ldots, 2 m\}$ such that ends $2 i-1$ and $2 i$ belong to edge $i$, for $1 \leq i \leq m$. In the edgecolouring area we place three exceptional cells for each end. These cells form a ' $t$ ' shape, which is illustrated in figure 3. (These shapes are apparent in figure 4.) Suppose end $i$ is incident with vertex $j$. We place the top-left cell of the ' $t$ ' shape for this end at position $(3 m+3 j-2,2 i-1)$. Each 't' shape will share its rows with two other 't'-shapes, because each vertex is incident with 3 ends.

In the bottom $2 m$ rows of the array we have a clean-up area. The exceptional cells are placed as $2 \times 3$ rectangles, as seen in the last 12 rows of figure 4 . This completes the description of $S(G)$. We will now look at some of its properties.

The reader can verify that $S(G)$ has been constructed so that there are exactly three exceptional cells in each column $i$, where $1 \leq i \leq 4 m$, and two exceptional cells in each column $i$, where $4 m+1 \leq i \leq n$. There are three exceptional cells in each row $j$, where $3 m+1 \leq j \leq n$. In rows $j$, where $1 \leq j \leq 3 m$, there is either one, two or three exceptional 


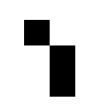

Figure 3: 't' shape

cells.

Lemma 5. Suppose we have a completion of $S(G)$. Then there is a set of three symbols such that every entry in an exceptional cell is from this set.

Proof. Without loss of generality we may assume that the exceptional cells in region $3 \mathrm{~m}$ have entries $\{1,2,3\}$. These cells are in row $3 m+1$, so clearly the exceptional cells in region $3 m+1$ (which are in row $3 m+2$ ) must have the same entries as those of region $3 m$. The same goes for the exceptional cells in region $3 m+2$, and all regions up to region $n$. Now, the exceptional cells in region $n$ are in rows 1 and 2. The entries in the exceptional cells in row 1 must be the same as the entries in the exceptional cells in region 1 (which are in row 3). Similarly the entry in the exceptional cell in row 2 must be the same as the entry in the exceptional cell in region 2 (which is in row 3 ). Hence the exceptional cells in row 3 contain the same entries as the exceptional cells in region $n$. As the entries in the exceptional cells in row 3 must be the same as the entries in the exceptional cells in region 3 , the pattern continues. Hence all the exceptional cells in the array must be filled with entries from $\{1,2,3\}$.

Lemma 6. Suppose we have a completion of $S(G)$. For all $i$, where $1 \leq i \leq m$, the entries in the exceptional cells in the edge-colouring area in columns $4 i-3$ and $4 i-1$ must be the same.

Proof. From lemma 5 we can assume that the exceptional cells all have entries from the set $\{1,2,3\}$. For all $i$, where $1 \leq i \leq m$, there are three exceptional cells in columns $4 i-3$ and $4 i-1$ - the first two of which are in rows $3 i-2$ and $3 i$. By the construction of $S(G)$, the two exceptional cells in row $3 i-2$ must have the same entries as the two exceptional cells in row $3 i$, although necessarily in a different order. This means that the remaining exceptional cell in column $4 i-3$ must have the same entry as the remaining exceptional cell in column $4 i-1$.

\section{Complexity of the decision problem}

Theorem 7. $S(G)$ has a completion if and only if the chromatic index of $G$ is 3.

Proof. Suppose $S(G)$ has a completion. By lemma 5 we can assume without loss of generality that all the exceptional cells have entries from the set $\{1,2,3\}$. We can colour the ends of the edges of $G$ using colours $\{1,2,3\}$ in the following way: end $i$ gets coloured according to the entry in the exceptional cell in column $2 i-1$ in the edge-colouring area. 
Now lemma 6 ensures that the two ends of an edge are coloured identically, so in fact our end-colouring is a proper edge-colouring using three colours. Hence the chromatic index of $G$ is 3 .

Conversely, suppose that the chromatic index of $G$ is 3 . So there exists a proper edge-colouring of $G$ using the colours $\{1,2,3\}$. This edge-colouring can be used to fill in the exceptional cells in rows $3 m+3 j-2$ for all $j$, where $1 \leq j \leq 2 m / 3$. We view the edge-colouring as an end-colouring, where the two ends of an edge are coloured identically. We can fill the cells in columns $2 i-1$ and $2 i$ according to the colour of the corresponding end $i$, where $1 \leq i \leq 2 m$. We then fill the other cells in the edge-colouring area according to the rule that in each ' $t$ '-shape all three entries from $\{1,2,3\}$ must occur. Clearly this can always be done in such a way that each row in the edge-colouring area has distinct entries in its exceptional cells. Next we fill the exceptional cells in the edge-setting area using $\{1,2,3\}$. They can clearly always be filled in such a way that the exceptional cells in each row and column contain distinct entries. The only remaining unfilled exceptional cells are in the clean-up area in the bottom-right of the array. We fill them with $\{1,2,3\}$ in any way that satisfies the row and column conditions.

Now all the exceptional cells have been filled. We now fill in some unexceptional cells with $\{1,2,3\}$. These will be in the top-right clean-up area, to the right of the edge-setting area. We will fill in cells so that every row and column of the array contains the entries $\{1,2,3\}$. Between rows $3 j-2$ and $3 j-1$, where $1 \leq j \leq m$, we are missing exactly the symbols $\{1,2,3\}$. The entries will be filled in cells in columns $4 m+3 j-2,4 m+3 j-1$ and $4 m+3 j$ - which symbol goes in which cell will depend on what is already in these columns. Clearly this can always be done.

So now the array has entries $\{1,2,3\}$ in each row and column. The exceptional cells have all been filled, so we can fill the remaining cells as if we were merely completing a uniform partial latin square. We now appeal to lemma 4 which shows that we can always complete the gerechte design.

The problem of deciding if a given gerechte skeleton has a completion is clearly in $\mathrm{NP}$, and we have shown that there is a polynomial transformation to it from a known NP-complete problem, namely the problem of deciding if cubic graphs have chromatic index 3. Thus we obtain theorems 1 and 2 .

\section{Complexity of the search problem}

Let us consider the complexity of finding a gerechte design for a given gerechte skeleton, which we shall call the "search problem". Suppose we had an oracle that could answer the decision problem from theorem 2 (which is NP-complete). Then we could solve the search problem by going through the $n^{2}$ cells in the array, at each stage trying all the symbols from $\{1, \ldots, n\}$ until the oracle says that the partial filling has a completion. Note that if the first call to the oracle gives a negative answer then the skeleton has no completion. The number of calls that need to be made to the oracle is obviously bounded by $n^{3}$ and so the computation can be carried out in polynomial time. 


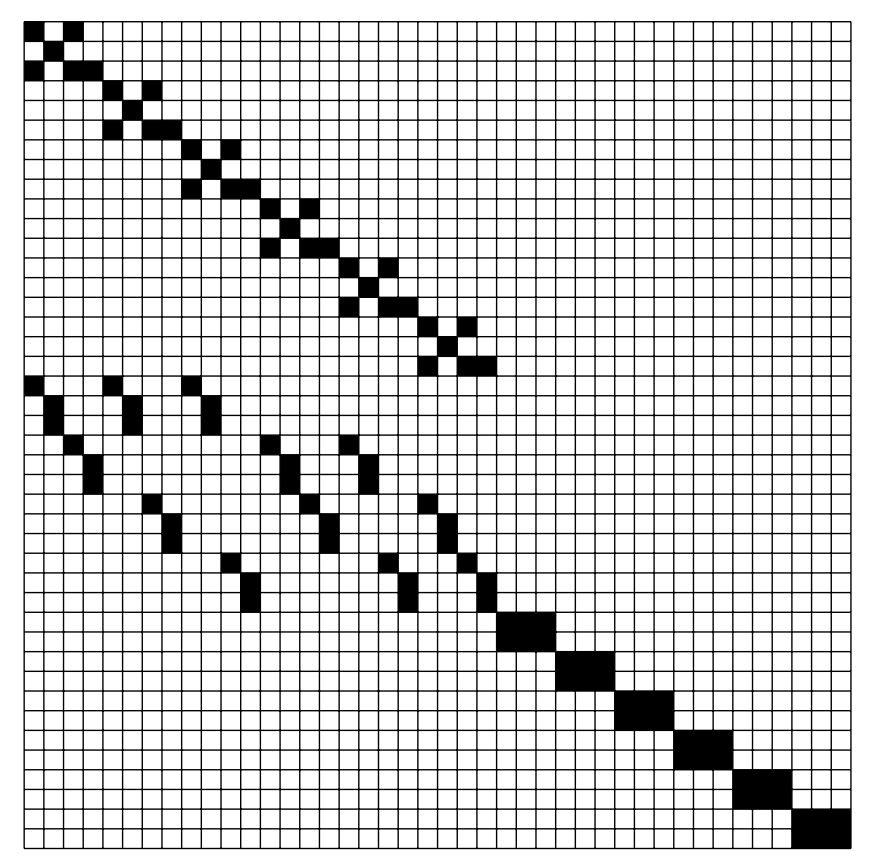

Figure 4: $S\left(K_{4}\right)$. The exceptional cells are drawn in black, the rest in white.

Thus the task of completing a gerechte skeleton is Turing-reducible to a problem in NP. This means that the search problem, while not in NP, is "no harder" than an NP-complete problem. It is from a class of problems that Garey and Johnson call "NP-equivalent". (This class is now commonly referred to as $\mathrm{FP}^{\mathrm{NP}}$ [9], although Garey and Johnson call it simply $\left.\mathrm{P}^{\mathrm{NP}}[7].\right)$

To conclude, there is a polynomial time algorithm for completing gerechte skeletons if and only if $\mathrm{P}=\mathrm{NP}$.

\section{$6 \quad$ Further questions}

Whilst the general problem of deciding if a given gerechte skeleton has a completion is NP-complete, we do not know what can be said about the computational complexity of the following subproblems.

Problem. Given a gerechte skeleton whose regions are contiguous, does it have a completion?

And:

Problem. Given a gerechte skeleton whose regions are rectangles, does it have a completion?

And (perhaps more importantly): 
Problem. Given a latin square, does it have an orthogonal mate?

This is indeed a subproblem of our main problem. Given a latin square, we can construct a gerechte skeleton by taking as its regions the sets of cells that contain particular symbols in the latin square. Completing this skeleton is equivalent to finding an orthogonal mate for the latin square.

\section{References}

[1] R. A. Bailey, Peter J. Cameron and Robert Connelly. Sudoku, gerechte designs, resolutions, affine space, spreads, reguli, and Hamming codes, Amer. Math. Monthly, 115 (2008), 383-404.

[2] W. U. Behrens, Feldversuchsanordnungen mit verbessertem Ausgleich der Bodenunterschiede, Zeitschrift für Landwirtschaftliches Versuchsund Untersuchungswesen, 2 (1956), 176-193.

[3] Peter J. Cameron (editor). Problems from CGCS Luminy, Europ. J. Combinatorics (submitted), 2007.

[4] C. J. Colbourn. Complexity of completing partial Latin squares. Discrete Appl. Math., 8 (1984), 25-30.

[5] Reinhard Diestel. Graph Theory, 3rd edition, Springer, 2005.

[6] T. Easton and R. Gary Parker. On completing latin squares. Discrete Appl. Math., 113 (2001), 167-181.

[7] Michael R. Garey and David S. Johnson. Computers and Intractability: A Guide to the Theory of NP-Completeness, W. H. Freeman, 1979.

[8] Ian Holyer. The NP-Completeness of Edge-Coloring, SIAM J. Comput., 10 (1981), $718-720$.

[9] Christos H. Papadimitriou. Computational Complexity, Addison Wesley, 1994. 